\title{
Wenn wir schon von Gerechtigkeit reden ...
}

\section{Samia Hurst}

Prof. Dr. med., Mitglied der Redaktion, Institut für Bioethik (iEH2), Medizinische Fakultät, Genf

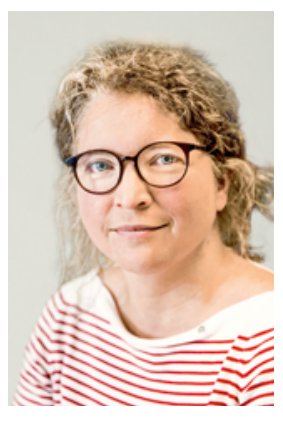

«Wo bleibt dabei die Verteilungsgerechtigkeit?» Wenn diese Frage in der Ethikdebatte auftaucht, werden die Blicke unstet. Gerechtigkeit zählt zu jenen wichtigen Werten, die häufig so schwierig in der Umsetzung sind. Was tun? Gerne greife ich hier wieder auf die Vorstellung einer «Ethik-Denkpause» bei einer Tasse Kaffee zurück, denn auch bei dieser Thematik verhelfen ein paar simple Argumentationspunkte zu einem klareren Blick. Sind Sie bereit? Los geht's!

1. Punkt: In der Medizin suchen wir nach mehr Gleichheit als in der Gesellschaft generell. In unseren Gesellschaften tolerieren wir offenkundig sehr viel Ungleichheit. Ganz eindeutig akzeptieren wir jedoch nicht, dass einige Menschen eine 200-fach bessere medizinische Behandlung erfahren als andere. Entspringt dieser Unterschied der Tatsache, dass wir uns unserer gemeinsamen Vulnerabilität bewusst sind, oder ist er das Resultat jener gesellschaftlichen Errungenschaft, die unser Gesundheitssystems ja darstellt? Die Klärung dieser Frage ist an dieser Stelle wenig relevant. Klar ist, dass der soziale Anspruch nach mehr Gleichheit stärker innerhalb als ausserhalb des Gesundheitswesens besteht. Für die Akteure im Gesundheitswesen heisst dies, dass der Zugang zum System nicht, oder nur so wenig wie möglich, durch Faktoren wie Status oder Finanzmittel des Patienten beeinflusst werden sollte. Gerechtigkeit im Gesundheitswesen ist eine ganz praktische, wenngleich auf hohem Niveau gestellte Forderung.

2. Punkt: Die Frage nach der Gerechtigkeit stellt sich in der Tat häufig, wenn Zweifel darüber bestehen, ob ein Eingriff begründet ist oder nicht. Nicht ein hoher finanzieller oder humaner Kostenaufwand lässt uns zögern, sondern vielmehr der Gedanke, ob sich der Eingriff lohnt oder nicht. Es muss daher geprüft werden, ob der Vorteil für den Patienten die Nachteile, die ihm erwachsen, überwiegt. Ist dies nicht der Fall, stehen wir nicht vor einer Gerechtigkeitsfrage, sondern vor der Frage nach vernünftigen Grenzen und therapeutischem Übereifer. Echte Gerechtigkeit hiesse, jeden Patienten nach seinen gesundheitlichen Bedürfnissen zu behandeln. Daraus ergeben sich mehrere koexistente Varianten: Soll das dafür erforderliche Vorgehen so gleich wie möglich gestaltet werden? Sollen zuerst die grössten Dringlichkeiten behandelt werden? Oder soll zuerst das behandelt werden, was dem Patienten die grösste Erleichterung verschafft? Im Extremfall erodiert die eine Variante die Möglichkeiten der anderen! Im Zweifelsfall lassen sie sich kombinieren mit dem Versuch, diese drei Arten von Ungerechtigkeiten so weit als möglich zu vermeiden [1]. In der Medizin ist die Problematik bekannt. Gelegentlich müssen Medikamente kombiniert werden, und das kann zur Folge haben, dass auch die Dosierung entsprechend angepasst werden muss. Das Ergebnis mag dann zwar nicht perfekt sein, aber es ist immer noch gut. Gerechtigkeit in der Medizin muss also nicht unbedingt perfekt sein, aber immer ausreichend. Eine wesentliche Komponente der ausreichenden Gerechtigkeit ist die Vermeidung von Diskriminierung. Es darf nicht mehr - oder weniger - zugeteilt werden wegen Kriterien, die nichts mit den Gesundheitsbedürfnissen zu tun haben. Alter kann unsere Bedürfnisse verändern, aber sie werden deshalb nicht weniger wichtig. Ein weiterer, wichtiger Aspekt in diesem Kontext ist die Vorgabe, dass Gerechtigkeit in der Medizin sich um faire Verteilungen kümmert und nie zu Strafe werden darf. Gelegentlich sind wir versucht, Patienten, denen wir die Verantwortung für ihre Erkrankung anlasten, als weniger wichtig einzustufen. Das ist jedoch nicht unsere Aufgabe.

Verteilungsgerechtigkeit in der Medizin bedeutet, dass wir uns dieser verschiedenen Aspekte bewusst sind und uns in unserem Handeln gewissen sozialen und persönlichen Vorbehalten widersetzen. Auch wenn sich Gerechtigkeit in einigen wenigen Punkten darstellen lässt, zählt sie doch in der Tat zu den sensibelsten Bereichen der Medizinethik. Um allzu subjektive Einstellungen zu vermeiden, bietet es sich an, Entscheide zu mehreren zu treffen, um sie so möglicherweise gerechter zu machen. Einige Ethiker sehen diesen Aspekt, die Verfahrensgerechtigkeit, sogar als notwendige Voraussetzung für Gleichheit [2]. In der Spitalarbeit ist die Beratung im Team ein Schritt in diese Richtung.

\section{Literatur}

1 Hurst SA, Mezger N, Mauron A. Allocating Resources in Humanitarian Medicine. Public Health Ethics. 2009;2(1):180-92.

2 Daniels N, Sabin JE. Accountability for reasonableness - an update. Brit Med J. 2008;337(7675). 